PROCEEDINGS OF THE

AMERICAN MATHEMATICAL SOCIETY

Volume 127, Number 11, Pages 3269-3277

S 0002-9939(99)05102-3

Article electronically published on May 4, 1999

\title{
VON NEUMANN ALGEBRAS AND LINEAR INDEPENDENCE OF TRANSLATES
}

PETER A. LINNELL

(Communicated by David R. Larson)

\begin{abstract}
For $x, y \in \mathbb{R}$ and $f \in L^{2}(\mathbb{R})$, define $(x, y) f(t)=e^{2 \pi i y t} f(t+x)$ and if $\Lambda \subseteq \mathbb{R}^{2}$, define $S(f, \Lambda)=\{(x, y) f \mid(x, y) \in \Lambda\}$. It has been conjectured that if $f \neq 0$, then $S(f, \Lambda)$ is linearly independent over $\mathbb{C}$; one motivation for this problem comes from Gabor analysis. We shall prove that $S(f, \Lambda)$ is linearly independent if $f \neq 0$ and $\Lambda$ is contained in a discrete subgroup of $\mathbb{R}^{2}$, and as a byproduct we shall obtain some results on the group von Neumann algebra generated by the operators $\{(x, y) \mid(x, y) \in \Lambda\}$. Also, we shall prove these results for the obvious generalization to $\mathbb{R}^{n}$.
\end{abstract}

\section{INTRODUCTION}

Let $n$ be a positive integer, let $\mathcal{G}_{n}$ be the abelian group $\left\{(x, y) \mid x, y \in \mathbb{R}^{n}\right\}$ with the operation addition (so $\mathcal{G}_{n} \cong \mathbb{R}^{2 n}$ ), and for $x, y \in \mathbb{R}^{n}$, let $x \cdot y$ denote the dot product $x_{1} y_{1}+\cdots+x_{n} y_{n}$. Let $\mathbb{C} * \mathcal{G}_{n}$ denote the twisted group ring (a twisted group ring is a particular kind of crossed product) which has $\mathbb{C}$-basis $\left\{\bar{g} \mid g \in \mathcal{G}_{n}\right\}$, and multiplication satisfying $\overline{(a, b)} \overline{(x, y)}=e^{2 \pi i a \cdot y} \overline{(a+x, b+y)}$. For $g \in \mathcal{G}_{n}$, we shall often write $g$ instead of $\bar{g}$ if there is no danger of confusion, and then $g^{-1}$ will mean $\bar{g}^{-1}$ rather than $\overline{g^{-1}}$. Let $L^{2}\left(\mathbb{R}^{n}\right)$ denote the Hilbert space of square integrable functions $\left\{f:\left.\mathbb{R}^{n} \rightarrow \mathbb{C}\left|\int_{\mathbb{R}^{n}}\right| f(t)\right|^{2} d t<\infty\right\}$ with two functions $f_{1}, f_{2} \in L^{2}\left(\mathbb{R}^{n}\right)$ being equal if and only if $f_{1}(t)=f_{2}(t)$ almost everywhere, and let $\mathcal{B}\left(L^{2}\left(\mathbb{R}^{n}\right)\right)$ denote the set of bounded linear operators on $L^{2}\left(\mathbb{R}^{n}\right)$. Then $\mathbb{C} * \mathcal{G}_{n}$ acts on the left of $L^{2}\left(\mathbb{R}^{n}\right)$ according to the rule $(x, y) f(t)=e^{2 \pi i y \cdot t} f(t+x)$ and extending to the whole of $\mathbb{C} * \mathcal{G}_{n}$ by $\mathbb{C}$-linearity. To check that this indeed defines an action, we need only verify that $(a, b)((x, y) f(t))=((a, b)(x, y)) f(t)$, which is indeed true because both sides equal $e^{2 \pi i(a \cdot y+b \cdot t+y \cdot t)} f(t+a+x)$. Thus we obtain a homomorphism from $\mathbb{C} * \mathcal{G}_{n}$ into $\mathcal{B}\left(L^{2}\left(\mathbb{R}^{n}\right)\right)$. Since $\mathbb{C} * \mathcal{G}_{n}$ is a simple ring by Lemma 2.1, this homomorphism must be a monomorphism and so we may view $\mathbb{C} * \mathcal{G}_{n}$ as a $\mathbb{C}$-subalgebra of $\mathcal{B}\left(L^{2}\left(\mathbb{R}^{n}\right)\right)$. We shall consider the following conjecture.

Conjecture 1.1. Let $0 \neq \theta \in \mathbb{C} * \mathcal{G}_{n}$ and $0 \neq f \in L^{2}\left(\mathbb{R}^{n}\right)$. Then $\theta f \neq 0$.

Motivation for studying this problem comes from Gabor analysis and in particular the conjecture on page 2790 of [4]. If $G \leqslant \mathcal{G}_{n}$, then $\mathbb{C} * G$ will denote the $\mathbb{C}$-subalgebra of $\mathbb{C} * \mathcal{G}_{n}$ which has $\mathbb{C}$-basis $\{\bar{g} \mid g \in G\}$. Of course when talking

Received by the editors January 30, 1998.

1991 Mathematics Subject Classification. Primary 46L10; Secondary 42C99.

Key words and phrases. Group von Neumann algebra, Gabor analysis, Heisenberg group. 
about discrete subsets of $\mathcal{G}_{n}$, we are giving $\mathcal{G}_{n}$ the usual topology from $\mathbb{R}^{2 n}$. We shall prove

Theorem 1.2. Let $G$ be a discrete subgroup of $\mathcal{G}_{n}$. If $0 \neq \theta \in \mathbb{C} * G$ and $0 \neq f \in$ $L^{2}\left(\mathbb{R}^{n}\right)$, then $\theta f \neq 0$.

Of course it follows immediately that if $G$ is a discrete subgroup of $\mathcal{G}_{n}, g \in \mathcal{G}_{n}$, $0 \neq \theta \in g \mathbb{C} * G$ and $0 \neq f \in L^{2}\left(\mathbb{R}^{n}\right)$, then $\theta f \neq 0$. This means we can rephrase the above result in terminology closer to that of [4] as follows. For $x, y \in \mathbb{R}^{n}$ and $f \in L^{2}\left(\mathbb{R}^{n}\right)$, define $(x, y) f(t)=e^{2 \pi i y \cdot t} f(t+x)$ and if $\Lambda \subseteq \mathbb{R}^{2 n}$, define $S(f, \Lambda)=$ $\{(x, y) f \mid(x, y) \in \Lambda\}$. Then Theorem 1.2 yields

Proposition 1.3. Let $n$ be a positive integer, let $\Lambda$ be a subset of $\mathbb{R}^{2 n}$ of the form $g+G$ where $G$ is a discrete subgroup of $\mathbb{R}^{2 n}$, and let $0 \neq f \in L^{2}\left(\mathbb{R}^{n}\right)$. Then $S(f, \Lambda)$ is linearly independent.

As a byproduct, we shall obtain results on the von Neumann algebra generated by $\mathbb{C} * G$, which we shall denote by $W * G$. Thus $W * G$ is the weak closure of $\mathbb{C} * G$ in $\mathcal{B}\left(L^{2}\left(\mathbb{R}^{n}\right)\right)$ and is rather similar to the group von Neumann algebra of $G$. For $f, g \in L^{2}\left(\mathbb{R}^{n}\right)$, let $\langle f, g\rangle$ denote the inner product $\int_{\mathbb{R}^{n}} f(t) \bar{g}(t) d t$, where ${ }^{-}$denotes complex conjugation, and let $\mathcal{U}\left(L^{2}\left(\mathbb{R}^{n}\right)\right)$ denote the set of closed densely defined linear operators $[5, \S 2.7]$ acting on $L^{2}\left(\mathbb{R}^{n}\right)$. Then the adjoint $\alpha^{*}$ of $\alpha \in \mathcal{U}\left(L^{2}\left(\mathbb{R}^{n}\right)\right)$ satisfies $\langle\alpha f, g\rangle=\left\langle f, \alpha^{*} g\right\rangle$ whenever $f, g \in L^{2}\left(\mathbb{R}^{n}\right)$ and $\alpha f, \alpha^{*} g$ are defined. Of course * restricts to an involution on both $\mathcal{B}\left(L^{2}\left(\mathbb{R}^{n}\right)\right)$ and $W * G$. If $G$ is a discrete subgroup of $\mathcal{G}_{n}$, then $W * G$ is a finite von Neumann algebra by Lemma 3.2; also in many cases this can be deduced from Rieffel's paper [7]. In this situation, we let $U * G$ indicate the operators of $\mathcal{U}\left(L^{2}\left(\mathbb{R}^{n}\right)\right)$ which are affiliated to $W * G[3$, p. 150]. The results of [3] (especially theorem 1 and the proof of theorem 10) now show that $(U * G)^{*}=U * G, U * G$ is a $*$-regular ring containing $W * G$, and every element of $U * G$ can be written in the form $\gamma \delta^{-1}$ where $\gamma, \delta \in W * G$. In particular every nonzero divisor in $W * G$ is invertible in $U * G$. We shall prove

Theorem 1.4. Let $G$ be a discrete subgroup of $\mathcal{G}_{n}$. Then $W * G$ is a finite von Neumann algebra, every nonzero element of $\mathbb{C} * G$ is invertible in $U * G$, and the set $\left\{\gamma \delta^{-1} \mid \gamma \in \mathbb{C} * G, 0 \neq \delta \in \mathbb{C} * G\right\}$ is a division subring of $U * G$.

Let $L$ be a locally compact group, let $G$ be a torsion free subgroup of $L$, and let $L^{2}(L)$ denote the Hilbert space of square integrable functions on $L$ with respect to the left Haar measure on $L$. Then $G$ acts on the left of $L^{2}(L)$ according to the rule $g f(l)=f\left(g^{-1} l\right)$ for $g \in G, f \in L^{2}(L), l \in L$. For $f \in L^{2}(L) \backslash 0$, a closely related problem to Conjecture 1.1 is to determine whether the set $\{g f \mid g \in G\}$ is linearly independent over $\mathbb{C}$. If the von Neumann algebra $W * G$ generated by $G$ is a finite von Neumann algebra, then by using the techniques of this paper, it is possible in many cases to show that the set $\{g f \mid g \in G\}$ is linearly independent. On the other hand if $W * G$ is not a finite von Neumann algebra, then the techniques of this paper cannot be applied. It will usually be the case that $W * G$ is not finite if $G$ is not discrete and has no abelian subgroup of finite index. A specific example would be to let $L$ be the Heisenberg group consisting of upper unitriangular 3 by 3 matrices with entries in $\mathbb{R}$, in other words matrices of the form

$$
\left(\begin{array}{ccc}
1 & a & b \\
0 & 1 & c \\
0 & 0 & 1
\end{array}\right)
$$


where $a, b, c \in \mathbb{R}$, and to let $G=L$. Then it is not known in this case whether for $f \in L^{2}(L) \backslash 0$, the set $\{g f \mid g \in G\}$ is linearly independent.

I am very grateful to Chris Heil and Jonathan Rosenblatt for bringing the problem studied in this paper to my attention, and for some helpful email correspondence.

\section{Notation, terminology AND ASSUmed RESUlts}

The identity of a group will be denoted by either 0 or 1 . If $n$ is a positive integer and $R$ is a ring, then $\mathrm{M}_{n}(R)$ will denote the $n$ by $n$ matrices over $R$, and we shall let $\delta_{i j}$ indicate the Kronecker delta, so $\delta_{i j}=0$ if $i \neq j$ and $\delta_{i j}=1$ if $i=j$. The identity matrix of $\mathrm{M}_{n}(R)$ will be denoted by $I_{n}$, and the zero matrix of $\mathrm{M}_{n}(R)$ will be denoted by $0_{n}$. We shall view vectors in $\mathbb{R}^{n}$ as column vectors rather than row vectors. A lattice in $\mathbb{R}^{n}$ will mean a discrete subgroup of $\mathbb{Z}$-rank $n$; in other words a discrete subgroup of finite covolume (note that this is a different definition of lattice from that of $\left[4\right.$, p. 2791]). If $\alpha=\sum_{g \in \mathcal{G}_{n}} \lambda_{g} g \in \mathbb{C} * \mathcal{G}_{n}$ where $\lambda_{g} \in \mathbb{C}$ for all $g \in \mathcal{G}_{n}$, then the support of $\alpha$, denoted $\operatorname{supp} \alpha$, is the set $\left\{g \in \mathcal{G}_{n} \mid \lambda_{g} \neq 0\right\}$. We shall use the notation $\|f\|_{2}$ for the norm $\sqrt{\langle f, f\rangle}$ of an element $f \in L^{2}\left(\mathbb{R}^{n}\right)$, and $\bar{X}$ for the closure of a subset $X$ in $L^{2}\left(\mathbb{R}^{n}\right)$. The commutant of a subset $A$ of $\mathcal{B}\left(L^{2}\left(\mathbb{R}^{n}\right)\right)$ is $A^{\prime}=\left\{x \in \mathcal{B}\left(L^{2}\left(\mathbb{R}^{n}\right)\right) \mid a x=x a\right.$ for all $\left.a \in A\right\}$. If $A=A^{*}$, then $A^{\prime}$ is a von Neumann algebra and by von Neumann's double commutant theorem [1, theorem 1.2.1], $A$ is dense in $A^{\prime \prime}$ in the weak operator topology. Thus another description of $W * G$ is the double commutant of $\mathbb{C} * G$ in $\mathcal{B}\left(L^{2}\left(\mathbb{R}^{n}\right)\right)$. In the case $W * G$ is a finite von Neumann algebra, we can now describe $U * G$ as those unbounded operators in $\mathcal{U}\left(L^{2}\left(\mathbb{R}^{n}\right)\right)$ which commute with every element of $(W * G)^{\prime}$.

Lemma 2.1. $\mathbb{C} * \mathcal{G}_{n}$ is a simple ring.

Proof. Suppose $0 \neq I \triangleleft \mathbb{C} * \mathcal{G}_{n}$ with $I \neq \mathbb{C} * \mathcal{G}_{n}$, and choose $0 \neq \alpha \in I$ with minimal support. If $g \in \operatorname{supp} \alpha$, then $1 \in \operatorname{supp} \bar{g}^{-1} \alpha$ and $\bar{g}^{-1} \alpha \in I$, so we may assume that $1 \in \operatorname{supp} \alpha$. Since $I \neq \mathbb{C} * \mathcal{G}_{n}$, we may choose $a \in \mathcal{G}_{n}$ such that $1 \neq a \in \operatorname{supp} \alpha$. Then there exists $g \in \mathcal{G}_{n}$ such that $\bar{g} \bar{a} \bar{g}^{-1} \neq \bar{a}$, and now we have $0 \neq \bar{g} \alpha \bar{g}^{-1}-\alpha \in I$. This contradicts the minimality of $\operatorname{supp} \alpha$ because $\left|\operatorname{supp}\left(\bar{g} \alpha \bar{g}^{-1}-\alpha\right)\right|<|\operatorname{supp} \alpha|$, and the result follows.

If $R$ is a ring and $\sigma$ is an automorphism of $R$, then $R_{\sigma}[X]$ will denote the twisted polynomial ring over $R$ in the indeterminate $X$, so multiplication is defined by $\sum a_{i} X^{i} \sum b_{j} X^{j}=\sum_{n}\left(\sum_{i+j=n} a_{i} \sigma^{i} b_{j}\right) X^{n}$. We say that $R$ is an Ore domain if it is contained in a division ring $D$, called the division ring of fractions of $R$, such that every element of $D$ can be written in the form $r s^{-1}$ and also in the form $s^{-1} r$, with $r, s \in R$ and $s \neq 0$. Of course the division ring $D$ containing $R$ is unique up to $R$-isomorphism. Also if $R$ is contained in a ring $D^{\prime}$ such that every nonzero element of $R$ is invertible, then the set $\left\{r s^{-1} \mid r, s \in R\right.$ and $\left.s \neq 0\right\}$ is the division ring of fractions containing $R$. The following two elementary results are well known.

Lemma 2.2. Let $R$ be an Ore domain with division ring of fractions $D$, and let $\sigma$ be an automorphism of $R$. Then $\sigma$ extends uniquely to an automorphism of $D$, which we shall also call $\sigma$, and if $\alpha, \beta \in D_{\sigma}[X]$, then there exists $r \in R \backslash 0$ such that $r \alpha, r \beta \in R_{\sigma}[X]$.

Lemma 2.3. Let $G$ be a subgroup of $\mathcal{G}_{n}$. Then $\mathbb{C} * G$ is an Ore domain, and if $I, J$ are nonzero left ideals of $\mathbb{C} * G$, then $I \cap J \neq 0$. 
Finally we require the following:

Lemma 2.4. Let $G$ be a discrete subgroup of $\mathcal{G}_{n}$, let $H \triangleleft G$ such that $G / H$ is infinite cyclic, and let $x \in G$ such that $H x$ is a generator for $G / H$. If $\zeta \in \mathbb{C}$ and $|\zeta|=1$, then there exists $y \in \mathcal{G}_{n}$ such that $\bar{y} \bar{h} \bar{y}^{-1}=\bar{h}$ for all $h \in H$ and $\bar{y} \bar{x} \bar{y}^{-1}=\zeta \bar{x}$ in $\mathbb{C} * \mathcal{G}_{n}$.

Proof. Since $G$ is discrete, we may choose $m \in \mathbb{Z}$ and a subset $\left\{h_{1}, \ldots, h_{m}\right\}$ which generates $H$ and is linearly independent over $\mathbb{R}$. Note that $\left\{h_{1}, \ldots, h_{m}, x\right\}$ is also linearly independent over $\mathbb{R}$. Choose $t \in \mathbb{R}$ such that $e^{2 \pi i t}=\zeta$, and define a bilinear form $\beta: \mathcal{G}_{n} \rightarrow \mathbb{R}$ by $\beta((a, b),(c, d))=a \cdot d-b \cdot c$, where $a, b, c, d \in \mathbb{R}^{n}$. Note that in $\mathbb{C} * \mathcal{G}_{n}$, we have

$$
(a, b)(c, d)(a, b)^{-1}=e^{2 \pi i(a \cdot d-b \cdot c)}(c, d) .
$$

It is easily checked that $\beta$ is nondegenerate, so there exists $y \in \mathcal{G}_{n}$ such that $\beta\left(y, h_{i}\right)=0$ for all $i$ and $\beta(y, x)=t$. This completes the proof.

\section{FAithFul TRACES}

In this section, we show that $W * G$ has a faithful weakly continuous tracial state, which in particular will establish that $W * G$ is a finite von Neumann algebra. Throughout this section, $n$ will be a positive integer. The purpose of the next lemma is to reduce to the case when $G$ is a lattice in $\mathbb{R}^{2 n}$ such that $G \cap 1 \times \mathbb{R}^{n}=1 \times \mathbb{Z}^{n}$; its proof is modelled on $[4, \S 2$, p. 2790].

We shall think of $\mathbb{R}^{2 n}$ as $\mathbb{R}^{n} \oplus \mathbb{R}^{n}$, so we can view $\mathbb{R}^{n}$ as a subgroup of $\mathbb{R}^{2 n}$ in the usual way via the map $x \mapsto(x, 0)$. We then have a monomorphism $\psi: \mathcal{G}_{n} \rightarrow \mathcal{G}_{2 n}$ and this induces a monomorphism $\mathbb{C} * \mathcal{G}_{n} \rightarrow \mathbb{C} * \mathcal{G}_{2 n}$, which we shall also call $\psi$.

Given $f, g \in L^{2}\left(\mathbb{R}^{n}\right)$, we can form the element $f \otimes g \in L^{2}\left(\mathbb{R}^{2 n}\right)$ defined by $(f \otimes g)(x, y)=f(x) g(y)$ for $x, y \in \mathbb{R}^{n}$, and then the functions of the form $\sum_{i=1}^{m} f_{i} \otimes g_{i}$ are dense in $L^{2}\left(\mathbb{R}^{2 n}\right)$. If $\theta \in \mathcal{B}\left(L^{2}\left(\mathbb{R}^{n}\right)\right)$, then we have a well defined operator $\theta \otimes 1 \in \mathcal{B}\left(L^{2}\left(\mathbb{R}^{2 n}\right)\right)$ satisfying $(\theta \otimes 1)(f \otimes g)=(\theta f) \otimes g$ for all $f, g \in L^{2}\left(\mathbb{R}^{n}\right)$, and this yields a weakly continuous $*$-monomorphism $\theta \mapsto \theta \otimes 1: \mathcal{B}\left(L^{2}\left(\mathbb{R}^{n}\right)\right) \rightarrow \mathcal{B}\left(L^{2}\left(\mathbb{R}^{2 n}\right)\right)$.

Note that when we view $\mathbb{C} * \mathcal{G}_{n}$ and $\mathbb{C} * \mathcal{G}_{2 n}$ as subalgebras of $\mathcal{B}\left(L^{2}\left(\mathbb{R}^{n}\right)\right)$ and $\mathcal{B}\left(L^{2}\left(\mathbb{R}^{2 n}\right)\right)$ respectively, then $\psi(\theta)=\theta \otimes 1$ for all $\theta \in \mathbb{C} * \mathcal{G}_{n}$. Furthermore, if $G \leq \mathcal{G}_{n}$, then $\psi$ induces isomorphisms $W * G \rightarrow W * \psi G$ and (assuming $W * G$ is a finite von Neumann algebra) $U * G \rightarrow U * \psi G$, which means we may identify $G$ with the subgroup $\psi G$ of $\mathcal{G}_{2 n}$; we shall do this without further comment and without using $\psi$ in the future.

Let $\left\{e_{1}, \ldots, e_{2 n}\right\}$ denote the standard basis for $\mathbb{R}^{2 n}$, so $e_{i}$ has a 1 in the $i$ th position and zeros elsewhere, and $e_{i} \cdot e_{j}=\delta_{i j}$. If $G \leqslant \mathcal{G}_{n}$, then we define $\{\mathbb{C} G\}=$ $\{\lambda g \mid \lambda \in \mathbb{C}$ and $g \in G\}$, a subset of $\mathbb{C} * G$.

Lemma 3.1. Let $G$ be a discrete subgroup of $\mathcal{G}_{n}$. Then there exists a lattice $H$ in $\mathcal{G}_{2 n}$ and a unitary operator $u \in \mathcal{B}\left(L^{2}\left(\mathbb{R}^{2 n}\right)\right)$, such that $H \cap 1 \times \mathbb{R}^{2 n}=1 \times$ $\mathbb{Z}^{2 n}, u\left\{\mathbb{C G}_{2 n}\right\} u^{-1}=\left\{\mathbb{C G}_{2 n}\right\}$ and $u\{\mathbb{C} G\} u^{-1} \subseteq\{\mathbb{C} H\}$.

Proof. Choose an $\mathbb{R}$-basis $\left\{g_{1}, \ldots, g_{2 n}\right\}$ for $\mathcal{G}_{n}$ such that $\left\{g_{1}, \ldots, g_{r}\right\}$ is a $\mathbb{Z}$-basis for $G$, where $r$ is the rank of $G$. Let $\mathcal{E}=\left\{\left(e_{1}, 0\right), \ldots,\left(e_{2 n}, 0\right),\left(0, e_{1}\right), \ldots,\left(0, e_{2 n}\right)\right\}$, 
let

$$
\begin{aligned}
\mathcal{F}=\left\{\left(e_{1}, e_{n+1}\right) / \sqrt{2},\left(e_{2}, e_{n+2}\right) / \sqrt{2}, \ldots,\left(e_{n}, e_{2 n}\right) / \sqrt{2},\left(e_{n+1}, e_{1}\right) / \sqrt{2},\right. \\
\left(e_{n+2}, e_{2}\right) / \sqrt{2}, \ldots,\left(e_{2 n}, e_{n}\right) / \sqrt{2},\left(-e_{n+1}, e_{1}\right) / \sqrt{2},\left(-e_{n+2}, e_{2}\right) / \sqrt{2}, \ldots, \\
\left.\left(-e_{2 n}, e_{n}\right) / \sqrt{2},\left(-e_{1}, e_{n+1}\right) / \sqrt{2},\left(-e_{2}, e_{n+2}\right) / \sqrt{2}, \ldots,\left(-e_{n}, e_{2 n}\right) / \sqrt{2}\right\},
\end{aligned}
$$

and let

$$
\begin{array}{r}
\mathcal{K}=\left\{g_{1}, \ldots, g_{2 n},\left(-e_{n+1}, e_{1}\right) / \sqrt{2},\left(-e_{n+2}, e_{2}\right) / \sqrt{2}, \ldots,\left(-e_{2 n}, e_{n}\right) / \sqrt{2},\right. \\
\left.\left(-e_{1}, e_{n+1}\right) / \sqrt{2},\left(-e_{2}, e_{n+2}\right) / \sqrt{2}, \ldots,\left(-e_{n}, e_{2 n}\right) / \sqrt{2}\right\},
\end{array}
$$

so $\mathcal{E}, \mathcal{F}$ and $\mathcal{K}$ are $\mathbb{R}$-bases of $\mathcal{G}_{2 n}$. For $i=1, \ldots, 4 n$, we shall let $\hat{e}_{i}, f_{i}, k_{i}$ denote the $i$ th basis elements of $\mathcal{E}, \mathcal{F}, \mathcal{K}$ respectively, and we shall let $K$ be the lattice in $\mathcal{G}_{2 n}$ which has $\mathbb{Z}$-basis $\mathcal{K}$. Let $A_{i}$ denote the coordinates of $k_{i}$ with respect to the basis $\mathcal{F}$, and let $a_{j i}$ denote the $j$ th coordinate of $A_{i}$. Then for $2 n+1 \leq i \leq 4 n$, $a_{j i}=1$ if $j=i$ and $a_{j i}=0$ if $j \neq i$. Now define $h_{i}=\sum_{j=1}^{4 n} a_{j i} \hat{e}_{j} \in \mathcal{G}_{2 n}$, and let $H$ be the subgroup of $\mathcal{G}_{2 n}$ generated by the $h_{i}$. Then $H$ is a lattice in $\mathcal{G}_{2 n}$ such that $H \cap 1 \times \mathbb{R}^{2 n}=1 \times \mathbb{Z}^{2 n}$.

Let $T$ be the transition matrix from $\mathcal{E}$ to $\mathcal{F}$, and let $J=J_{2 n}=\left(\begin{array}{cc}0_{n} & I_{n} \\ I_{n} & 0_{n}\end{array}\right) \in$ $\mathrm{M}_{2 n}(\mathbb{R})$. Thus if $T$ has entries $t_{i j}$, then $f_{j}=\sum_{i=1}^{4 n} t_{i j} \hat{e}_{i}$, and if we think of the $A_{i}$ as column vectors, then the coordinates of $k_{i}$ with respect to $\mathcal{E}$ are $T A_{i}$. Also

$$
\begin{aligned}
T & =\left(\begin{array}{cc}
I_{2 n} / \sqrt{2} & -J_{2 n} / \sqrt{2} \\
J_{2 n} / \sqrt{2} & I_{2 n} / \sqrt{2}
\end{array}\right) \\
& =\left(\begin{array}{cc}
I_{2 n} & 0_{2 n} \\
-J_{2 n} & I_{2 n}
\end{array}\right)\left(\begin{array}{cc}
J_{2 n} / \sqrt{2} & 0_{2 n} \\
0_{2 n} & J_{2 n} \sqrt{2}
\end{array}\right)\left(\begin{array}{cc}
0_{2 n} & -I_{2 n} \\
I_{2 n} & 0_{2 n}
\end{array}\right)\left(\begin{array}{cc}
I_{2 n} & 0_{2 n} \\
-J_{2 n} & I_{2 n}
\end{array}\right) .
\end{aligned}
$$

Let $\tau, \alpha, \beta, \gamma: \mathcal{G}_{2 n} \rightarrow \mathcal{G}_{2 n}$ be the linear mappings determined by the matrices

$$
T,\left(\begin{array}{cc}
J_{2 n} / \sqrt{2} & 0_{2 n} \\
0_{2 n} & J_{2 n} \sqrt{2}
\end{array}\right),\left(\begin{array}{cc}
I_{2 n} & 0_{2 n} \\
-J_{2 n} & I_{2 n}
\end{array}\right),\left(\begin{array}{cc}
0_{2 n} & -I_{2 n} \\
I_{2 n} & 0_{2 n}
\end{array}\right)
$$

respectively, with respect to the basis $\mathcal{E}$, so $\tau \hat{e}_{i}=f_{i}$ for all $i$. Then $\tau H=K \supseteq G$, so it will be sufficient to show that there exists a unitary operator $u \in \mathcal{B}\left(L^{2}\left(\mathbb{R}^{2 n}\right)\right)$ such that $u^{-1} \mathbb{C} g u=\mathbb{C} \tau g$ for all $g \in \mathcal{G}_{2 n}$. Since $\tau=\beta \alpha \gamma \beta$, it will be sufficient to do this with $\alpha, \beta, \gamma$ in place of $\tau$. We now use metaplectic transformations [8, p. 578]. Write $g=(x, y)$ where $x, y \in \mathbb{R}^{2 n}$, and then we have three cases to consider.

1. The matrix $\alpha=\left(\begin{array}{cc}J_{2 n} / \sqrt{2} & 0_{2 n} \\ 0_{2 n} & \sqrt{2} J_{2 n}\end{array}\right)$. For $f \in L^{2}\left(\mathbb{R}^{2 n}\right)$ and $t \in \mathbb{R}^{2 n}$, we define $u f(t)=2^{-n / 2} f\left(J t / \sqrt{2}\right.$ ) (we are considering $t$ as a column vector in $\mathbb{R}^{2 n}$ here). Then $u$ is $\mathbb{C}$-linear and $\|u f\|_{2}=\|f\|_{2}$ for all $f \in L^{2}\left(\mathbb{R}^{2 n}\right)$, hence $u$ is a unitary operator. Also $u^{-1} f(t)=2^{n / 2} f(\sqrt{2} J t)$ because $J_{2 n}^{2}=I_{2 n}$, consequently

$$
\begin{aligned}
u^{-1} g u f(t) & =u^{-1} g 2^{-n / 2} f(J t / \sqrt{2})=u^{-1} e^{2 \pi i y \cdot t} 2^{-n / 2} f(J(t+x) / \sqrt{2}) \\
& =e^{2 \pi i \sqrt{2} J y \cdot t} f(t+J x / \sqrt{2}) \quad \text { because } J_{2 n} \text { is symmetric } \\
& =(\alpha g) f(t)
\end{aligned}
$$

for all $t \in \mathbb{R}^{2 n}$ and for all $f \in L^{2}\left(\mathbb{R}^{2 n}\right)$. Thus $u^{-1} g u=\alpha g$ as required. 
2. The matrix $\beta=\left(\begin{array}{cc}I_{2 n} & 0_{2 n} \\ -J_{2 n} & I_{2 n}\end{array}\right)$. Here we define $u f(t)=e^{-\pi i J t \cdot t} f(t)$. Then $u$ is $\mathbb{C}$-linear and $\|u f\|_{2}=\|f\|_{2}$, so $u$ is a unitary operator. Since $u^{-1} f(t)=$ $e^{\pi i J t \cdot t} f(t)$,

$$
\begin{aligned}
u^{-1} g u f(t) & =u^{-1} g e^{-\pi i J t \cdot t} f(t)=u^{-1} e^{2 \pi i y \cdot t} e^{-\pi i J(t+x) \cdot(t+x)} f(t+x) \\
& =e^{\pi i J t \cdot t} e^{2 \pi i y \cdot t} e^{-\pi i J(t+x) \cdot(t+x)} f(t+x) \\
& =e^{-\pi i J x \cdot x} e^{2 \pi i t \cdot(y-J x)} f(t+x) \quad \text { because } J_{2 n} \text { is symmetric } \\
& =e^{-\pi i J x \cdot x}(\beta g) f(t)
\end{aligned}
$$

for all $t \in \mathbb{R}^{2 n}$, and we have shown that $u^{-1} g u \in \mathbb{C}(\beta g)$.

3. The matrix $\gamma=\left(\begin{array}{cc}0_{2 n} & -I_{2 n} \\ I_{2 n} & 0_{2 n}\end{array}\right)$. Here we use the Fourier transform; specifically $u f(t)=\int_{\mathbb{R}^{2 n}} e^{2 \pi i t \cdot s} f(s) d s$, and then $u^{-1} f(t)=\int_{\mathbb{R}^{2 n}} e^{-2 \pi i t \cdot s} f(s) d s$. Observe that $(x, 0) u f(t)=u(0, x) f(t)$ and $(0, y) u f(t)=u(-y, 0) f(t)$, consequently

$u^{-1}(x, y) u=u^{-1}(0, y)(x, 0) u=(-y, 0)(0, x)=e^{-2 \pi i x \cdot y}(-y, x)=e^{-2 \pi i x \cdot y} \gamma(x, y)$ and we deduce that $u^{-1} \mathbb{C} g u=\mathbb{C} \gamma g$.

This completes the proof of Lemma 3.1

Lemma 3.2. Let $G$ be a discrete subgroup of $\mathcal{G}_{n}$, and define $\tau: \mathbb{C} * G \rightarrow \mathbb{C}$ by $\tau g=0$ when $1 \neq g \in G$, and $\tau 1=1$. Then

(i) $\tau$ extends to a weakly continuous $\mathbb{C}$-linear map $W * G \rightarrow \mathbb{C}$.

(ii) If $\alpha, \beta \in W * G$, then $\tau(\alpha \beta)=\tau(\beta \alpha)$.

(iii) If $\alpha \in W * G$ and $x \in \mathcal{G}_{n}$, then $\tau\left(\bar{x} \alpha \bar{x}^{-1}\right)=\tau(\alpha)$.

(iv) If $e$ is a nonzero projection in $W * G$, then $0<\tau e \leq 1$.

(v) Let $e, f$ be projections in $W * G$. If $e L^{2}\left(\mathbb{R}^{n}\right) \subseteq f L^{2}\left(\mathbb{R}^{n}\right)$, then $\tau e \leq \tau f$.

(vi) Let $e, f$ be projections in $W * G$, and let $h$ be the projection of $L^{2}\left(\mathbb{R}^{n}\right)$ onto $\overline{e L^{2}\left(\mathbb{R}^{n}\right)+f L^{2}\left(\mathbb{R}^{n}\right)}$. Then $h \in W * G$ and if $e L^{2}\left(\mathbb{R}^{n}\right) \cap f L^{2}\left(\mathbb{R}^{n}\right)=0$, then $\tau e+\tau f=\tau h$.

Proof. Since $G$ is a discrete subgroup of $\mathcal{G}_{n}$, there is by Lemma 3.1, a lattice $H$ in $\mathcal{G}_{2 n}$ and a unitary operator $u \in \mathcal{B}\left(L^{2}\left(\mathbb{R}^{2 n}\right)\right)$ such that $H \cap 1 \times \mathbb{R}^{2 n}=1 \times$ $\mathbb{Z}^{2 n}, u\left\{\mathbb{C G}_{2 n}\right\} u^{-1}=\left\{\mathbb{C G}_{2 n}\right\}$ and $u \mathbb{C} * G u^{-1} \subseteq \mathbb{C} * H$. If we can find a weakly continuous $\mathbb{C}$-linear map $\tau: \mathbb{C} * H \rightarrow \mathbb{C}$ with the required properties, then the weakly continuous $\mathbb{C}$-linear map $\alpha \mapsto \tau\left(u \alpha u^{-1}\right)$ for $\alpha \in \mathbb{C} * G$ will suffice. Therefore we may assume that $G$ is a lattice in $\mathcal{G}_{n}$ and $G \cap 1 \times \mathbb{R}^{n}=1 \times \mathbb{Z}^{n}$. If $a$ is a positive number, we shall let $\mathcal{C}(a)$ denote the standard unit cube in $\mathbb{R}^{n}$ with side of length $a$; thus $\mathcal{C}(a)=\left\{\left(a_{1}, \ldots, a_{n}\right) \mid 0 \leq a_{i} \leq a\right.$ for all $\left.i\right\}$.

(i) Choose a positive integer $b$ such that $h \mathcal{C}(1 / b) \cap \mathcal{C}(1 / b)=\emptyset$ whenever $(h, k) \in$ $G \backslash\left(1 \times \mathbb{Z}^{n}\right)$, which is possible because $G$ is a lattice in $\mathcal{G}_{n}$ such that $G \cap 1 \times \mathbb{R}^{n}=$ $1 \times \mathbb{Z}^{n}$, and set $\mathcal{C}=\mathcal{C}(1 / b)$. Let $c=b^{n}$, let $\mathcal{C}_{1}, \ldots, \mathcal{C}_{c}$ denote the $c$ translates of $\mathcal{C}$ which are contained in the unit cube $\mathcal{C}(1)$, and for each $i$, let $\chi_{i}$ denote the characteristic function of $\mathcal{C}_{i}$. For $\theta \in W * G$, define

$$
\tau \theta=\sum_{i=1}^{c}\left\langle\theta \chi_{i}, \chi_{i}\right\rangle=\sum_{i=1}^{c} \int_{\mathcal{C}_{i}} \theta \chi_{i}(t) d t .
$$

Let $g \in G$ and write $g=(h, k)$ where $h, k \in \mathbb{R}^{n}$. Then $g \chi_{i}(t)=e^{2 \pi i k \cdot t} \chi_{i}(t+h)$, so if $h \neq 0$ we have $g \chi_{i}(t)=0$ for all $t \in \mathcal{C}_{i}$ and hence $\tau g=0$. On the other hand if 
$h=0$ and $k \neq 0$, then $k \in \mathbb{Z}^{n} \backslash 0$ because $G$ is a lattice such that $G \cap 1 \times \mathbb{R}^{n}=1 \times \mathbb{Z}^{n}$, consequently

$$
\tau g=\sum_{i=1}^{c} \int_{\mathcal{C}_{i}} e^{2 \pi i k \cdot t} d t=\int_{\mathcal{C}} e^{2 \pi i k \cdot t} d t=0
$$

Finally $\tau 1=1$ and (i) is proven.

(ii) If $x, y \in G$, then $x y=1$ if and only if $y x=1$. Therefore $\tau \bar{x} \bar{y}=\tau \bar{y} \bar{x}=0$ if $x y \neq 1$ and $\bar{x} \bar{y}=\bar{y} \bar{x}$ if $x y=1$, hence $\tau \bar{x} \bar{y}=\tau \bar{y} \bar{x}$ for all $x, y \in G$ and we deduce that $\tau \alpha \beta=\tau \beta \alpha$ for all $\alpha, \beta \in \mathbb{C} * G$. Since $\tau$ is weakly continuous and $W * G$ is the weak closure of $\mathbb{C} * G$, we see that $\tau \alpha \beta=\tau \beta \alpha$ for all $\alpha, \beta \in W * G$, which proves (ii).

(iii) Define $\sigma: W * G \rightarrow \mathbb{C}$ by $\sigma(\alpha)=\tau\left(x \alpha x^{-1}\right)$. Observe that $\sigma 1=1$ and if $1 \neq g \in G$, then $\sigma g=\tau\left(x g x^{-1}\right)=0$ because $x g x^{-1}=\zeta g$ for some $\zeta \in \mathbb{C}$ with $|\zeta|=1$. Thus $\sigma g=\tau g$ for all $g \in G$ and since $\sigma$ is a weakly continuous $\mathbb{C}$-linear map, we deduce that $\sigma(\alpha)=\tau(\alpha)$ for all $\alpha \in W * G$, which is the required result.

(iv) Note that if $e$ is a projection in $W * G$, then $e^{*} e=e$, and hence

$$
\tau e=\sum_{i=1}^{c}\left\langle e^{*} e \chi_{i}, \chi_{i}\right\rangle=\sum_{i=1}^{c}\left\langle e \chi_{i}, e \chi_{i}\right\rangle \geq 0 .
$$

Let $K=\mathbb{Z}^{n} \times \mathbb{Z}^{n} \leqslant \mathcal{G}_{n}$. If $k \in K$, then $\tau e=\tau\left(k^{-1} e k\right)$ by (iii) and we deduce that

$$
\tau e=\tau\left(k^{-1} e k\right)=\sum_{i=1}^{c}\left\langle k^{-1} e^{*} e k \chi_{i}, \chi_{i}\right\rangle=\sum_{i=1}^{c}\left\langle e k \chi_{i}, e k \chi_{i}\right\rangle .
$$

Let $\chi$ denote the characteristic function of $\mathcal{C}(1)$, and suppose $\tau e=0$. Then $e k \chi_{i}=0$ for all $i$, hence $e k \chi=0$ for all $k \in K$. Now the set $\{k \chi \mid k \in K\}$ forms a Hilbert basis for $L^{2}\left(\mathbb{R}^{n}\right)$ so if $\tau e=0$, we see that $e f=0$ for all $f \in L^{2}\left(\mathbb{R}^{n}\right)$ and we deduce that $e=0$. Also $1-e$ is a projection if $e$ is a projection, so applying the above to $1-e$ we obtain $0 \leq \tau(1-e)$, hence $\tau e \leq 1$ and (iv) follows.

(v) Let $h$ be the projection of $L^{2}\left(\mathbb{R}^{n}\right)$ onto the orthogonal complement of $e L^{2}\left(\mathbb{R}^{n}\right)$ in $f L^{2}\left(\mathbb{R}^{n}\right)$. Then $e+h=f$, hence $\tau e+\tau h=\tau f$. Thus $h \in W * G$ and the result follows from (iv).

(vi) Let $u$ be a unitary operator in $(\mathbb{C} * G)^{\prime}$. Then $u e u^{-1}=e$ and $u f u^{-1}=f$. Since $h$ is the projection of $L^{2}\left(\mathbb{R}^{n}\right)$ onto $\overline{e L^{2}\left(\mathbb{R}^{n}\right)+f L^{2}\left(\mathbb{R}^{n}\right)}$, we see that $u h u^{-1}$ is the projection of $L^{2}\left(\mathbb{R}^{n}\right)$ onto $\overline{u e u^{-1} L^{2}\left(\mathbb{R}^{n}\right)+u f u^{-1} L^{2}\left(\mathbb{R}^{n}\right)}=\overline{e L^{2}\left(\mathbb{R}^{n}\right)+f L^{2}\left(\mathbb{R}^{n}\right)}$ and we deduce that $u h u^{-1}=h$. Therefore $u h=h u$. Now $(\mathbb{C} * G)^{\prime}$ is a von Neumann algebra, so any element of $(\mathbb{C} * G)^{\prime}$ is a $\mathbb{C}$-linear sum of unitary elements, hence $x h=h x$ for all $x \in(\mathbb{C} * G)^{\prime}$ and we conclude that $h \in W * G$.

We now claim that $h=e \cup f\left[2\right.$, p. 4]. Since $e L^{2}\left(\mathbb{R}^{n}\right) \subseteq h L^{2}\left(\mathbb{R}^{n}\right)$, we see that $e=h e$ and hence $e \leq h$. Similarly $f \leq h$ and so $e \cup f \leq h$. Now let $g=e \cup f$. Then $g L^{2}\left(\mathbb{R}^{n}\right) \supseteq e L^{2}\left(\mathbb{R}^{n}\right), f L^{2}\left(\mathbb{R}^{n}\right)$ and hence $g L^{2}\left(\mathbb{R}^{n}\right) \supseteq h L^{2}\left(\mathbb{R}^{n}\right)$. We deduce that $g \geq h$, consequently $g=h$ and the claim is established.

If $e L^{2}\left(\mathbb{R}^{n}\right) \cap f L^{2}\left(\mathbb{R}^{n}\right)=0$, then $(e \cap f) L^{2}\left(\mathbb{R}^{n}\right) \subseteq e L^{2}\left(\mathbb{R}^{n}\right) \cap f L^{2}\left(\mathbb{R}^{n}\right)=0$. Since $W * G$ is a von Neumann algebra, we may apply the parallelogram law to deduce that $e \sim e \cup f-f[2, \S 1, \S 13]$. Thus there is an element $w \in W * G$ such that $w^{*} w=e$ and $w w^{*}=e \cup f-f$. Since $\tau\left(w^{*} w\right)=\tau\left(w w^{*}\right)$, we deduce that $\tau e=\tau(e \cup f-f)$ and hence $\tau e+\tau f=\tau(e \cup f)=\tau h$. This completes the proof. 


\section{Proofs}

Theorems 1.2 and 1.4 are now immediate consequences of the following result.

Lemma 4.1. Let $G$ be a discrete subgroup of $\mathbb{R}^{n}$ and let $\theta \in \mathbb{C} * G \backslash 0$. Then

(i) If $0 \neq f \in L^{2}\left(\mathbb{R}^{n}\right)$, then $\theta f \neq 0$.

(ii) $\theta$ is invertible in $U * G$.

(iii) The set $\left\{\gamma \delta^{-1} \mid \gamma \in \mathbb{C} * G, 0 \neq \delta \in \mathbb{C} * G\right\}$ is a division subring of $U * G$, and is equal to $\left\{\delta^{-1} \gamma \mid \gamma \in \mathbb{C} * G, 0 \neq \delta \in \mathbb{C} * G\right\}$.

Proof. Since $G$ is a discrete subgroup of $\mathcal{G}_{n}$, it is a free abelian group of rank at most $2 n$. We shall prove the result by induction on the rank of $G$, the result being trivially true if the rank of $G$ is zero, because then $G=1$. Thus we may assume that the rank of $G$ is strictly positive, and then there exists $H \triangleleft G$ such that $G / H \cong \mathbb{Z}$. Since $H$ has strictly smaller rank than $G$, we may assume that the result is true for $H$. Let $\tau: W * G \rightarrow \mathbb{C}$ be the weakly continuous tracial state obtained from Lemma 3.2.

(i) For $\alpha \in \mathbb{C} * G$, let $\operatorname{ker} \alpha=\left\{f \in L^{2}\left(\mathbb{R}^{n}\right) \mid \alpha f=0\right\}$, and let $\mathcal{N}(\alpha)$ be the projection from $L^{2}\left(\mathbb{R}^{n}\right)$ onto ker $\alpha$. Suppose $u$ is a unitary element in $(\mathbb{C} * G)^{\prime}$. Then $u^{-1} \mathcal{N}(\alpha) u=\mathcal{N}\left(u^{-1} \alpha u\right)=\mathcal{N}(\alpha)$. Since $(\mathbb{C} * G)^{\prime}$ is a von Neumann algebra, every element of $(\mathbb{C} * G)^{\prime}$ is a linear combination of unitary elements of $(\mathbb{C} * G)^{\prime}$ and we deduce that $\mathcal{N}(\alpha)$ commutes with every element of $(\mathbb{C} * G)^{\prime}$. Therefore $\mathcal{N}(\alpha) \in W * G$.

Let $\nu=\sup \{\tau(\mathcal{N}(\alpha)) \mid 0 \neq \alpha \in \mathbb{C} * G\}$. If $\nu=0$, then $\mathcal{N}(\theta)=0$ by Lemma 3.2(iv), hence $\operatorname{ker} \theta=0$ and the result follows, so we may assume that $0<\nu \leq 1$. Therefore we may choose $\alpha \in \mathbb{C} * G$ such that $\tau \mathcal{N}(\alpha)>\nu / 2$. Since $G / H$ is infinite cyclic, there exists $x \in G$ such that $H x$ generates $G / H$, and then we may write $\alpha=\sum_{i=-\infty}^{i=\infty} \alpha_{i} x^{i}$ where $\alpha_{i} \in \mathbb{C} * H$ and $\alpha_{i}=0$ for all but finitely many $i$. By replacing $\alpha$ with $x^{m} \alpha$ for some integer $m$, we may assume that $\alpha_{0} \neq 0$ and $\alpha_{i}=0$ for all $i<0$.

By induction, there is a division subring $D$ of $U * H$ containing $\mathbb{C} * H$ which is the division ring of fractions of $\mathbb{C} * H$. Let $\sigma$ be the automorphism $\beta \mapsto x \beta x^{-1}: \mathbb{C} * H \rightarrow$ $\mathbb{C} * H$. By Lemma 2.2 we may extend $\sigma$ to an automorphism of $D$, which we shall also call $\sigma$. We now have a natural ring homomorphism $\theta: D_{\sigma}[X] \rightarrow U * G$, defined by $\theta X=x$ and $\theta d=d$ for all $d \in D$, which maps $(\mathbb{C} * H)_{\sigma}[X]$ into $\mathbb{C} * G$. By $[6$, lemma 16], there exists $\zeta \in \mathbb{C}$ with $|\zeta|=1$ and $\beta^{\prime}, \gamma^{\prime} \in D_{\sigma}[X]$ such that

$$
\beta^{\prime} \sum_{i} \alpha_{0}^{-1} \alpha_{i} X^{i}+\gamma^{\prime} \sum_{i} \alpha_{0}^{-1} \alpha_{i} \zeta^{i} X^{i}=1 .
$$

By Lemma 2.2, there exists $0 \neq r \in \mathbb{C} * H$ such that $r \beta^{\prime} \alpha_{0}^{-1}, r \gamma^{\prime} \alpha_{0}^{-1} \in(\mathbb{C} * H)_{\sigma}[X]$, so setting $\beta=r \beta^{\prime} \alpha_{0}^{-1}$ and $\gamma=r \gamma^{\prime} \alpha_{0}^{-1}$, we have $\beta, \gamma \in(\mathbb{C} * H)_{\sigma}[X]$ and

$$
\beta \sum_{i} \alpha_{i} X^{i}+\gamma \sum_{i} \alpha_{i} \zeta^{i} X^{i}=r
$$

Set $\alpha^{\prime}=\sum_{i} \alpha_{i} \zeta^{i} x^{i}$. Applying the homomorphism $\theta$, we now have $\beta \alpha+\gamma \alpha^{\prime}=r$. By Lemma 2.4 there exists $y \in \mathcal{G}_{n}$ such that $y h y^{-1}=h$ for all $h \in H$ and $y x y^{-1}=\zeta x$, and then we have $y \alpha y^{-1}=\alpha^{\prime}$. Thus $\operatorname{ker} \alpha^{\prime}=y(\operatorname{ker} \alpha) y^{-1}$, consequently $\mathcal{N}\left(\alpha^{\prime}\right)=$ $y \mathcal{N}(\alpha) y^{-1}$ and using Lemma 3.2(iii), we deduce that $\tau \mathcal{N}\left(\alpha^{\prime}\right)=\tau N(\alpha)>\nu / 2$.

Suppose $f \in \operatorname{ker} \alpha \cap \operatorname{ker} \alpha^{\prime}$. Then $\alpha f=\alpha^{\prime} f=0$, hence $r f=0$ because $r=$ $\beta \alpha+\gamma \alpha^{\prime}$, and we can invoke our inductive hypothesis to deduce that $f=0$. 
Therefore $\operatorname{ker} \alpha \cap \operatorname{ker} \alpha^{\prime}=0$. If $\pi$ is the projection onto $\overline{\operatorname{ker} \alpha+\operatorname{ker} \alpha^{\prime}}$, we now see from Lemma 3.2(vi) that

$$
\tau \pi=\tau \mathcal{N}(\alpha)+\tau \mathcal{N}\left(\alpha^{\prime}\right)>\nu / 2+\nu / 2=\nu .
$$

Using Lemma 2.3 we may choose $\delta$ so that $0 \neq \delta \in \mathbb{C} * G \alpha \cap \mathbb{C} * G \alpha^{\prime}$, and then $\operatorname{ker} \delta \supseteq \operatorname{ker} \alpha+\operatorname{ker} \alpha^{\prime}$, hence $\tau \mathcal{N}(\delta) \geq \tau \pi>\nu$ by Lemma 3.2(v). This contradicts the definition of $\nu$ and (i) is proven.

(ii) This follows from (i) and the remarks immediately preceding Theorem 1.4.

(iii) This follows from (ii), Lemma 2.3 and the comments immediately preceding Lemma 2.2 .

\section{REFERENCES}

1. W. Arveson, An invitation to $C^{*}$-algebra, Graduate Texts in Mathematics, vol. 39, SpringerVerlag, Berlin-New York, 1976. MR 58:23621

2. S. K. Berberian, Baer *-rings, Grundlehren, vol. 195, Springer-Verlag, Berlin-New York, 1972. MR 55:2983

3. , The maximal ring of quotients of a finite von Neumann algebra, Rocky Mountain J. Math. 12 (1982), 149-164. MR 83i:16005

4. C. Heil, J. Ramanathan, and P. Topiwala, Linear independence of time-frequency translates, Proc. Amer. Math. Soc. 124 (1996), 2787-2795. MR 96k:42039

5. R. V. Kadison and J. R. Ringrose, Fundamentals of the theory of operator algebras, volume 1, elementary theory, Pure and Applied Mathematics Series, vol. 100, Academic Press, LondonNew York, 1983. MR 85j:46099

6. P. A. Linnell, Zero divisors and group von Neumann algebras, Pacific J. Math. 149 (1991), 349-363. MR 92e:22013

7. Marc A. Rieffel, Von Neumann algebras associated with pairs of lattices in Lie groups, Math. Ann. 257 (1981), 403-418. MR 84f:22010

8. E. M. Stein, Harmonic analysis: real-variable methods, orthogonality, and oscillatory integrals, Princeton Mathematical Series, vol. 43, Princeton University Press, Princeton, N.J., 1993. MR 95c: 42002

Department of Mathematics, Virginia Polytech Institute and State University, Blacksburg, Virginia 24061-0123

E-mail address: linnell@math.vt.edu

URL: http://www.math.vt.edu/people/linnell/ 